\title{
A modified presurgical alveolar molding technique for treatment of cleft in Down syndrome
}

Merve Gonca ${ }^{\mathrm{a}}$ (1)

Mehmet Birol Ozel ${ }^{\mathrm{b}}$

${ }^{a}$ Department of Orthodontics, Faculty of Dentistry, Recep Tayyip Erdoğan University, Rize, Turkey

${ }^{b}$ Department of Orthodontics, Faculty of Dentistry, Kocaeli University, Kocaeli, Turkey
Craniofacial clefts are extremely rare deformities. Tessier's classification is a widely accepted system that is based on clinical, radiographical, and surgical observations. The Tessier No. 0 cleft most commonly affects the upper lip, nose, and palate. This case presentation aims to report the outcome of a modified presurgical alveolar molding (PAM) appliance used in the treatment of an infant with Tessier No. 0 cleft as an alternate approach to mold such defects before surgery. The modified PAM appliance consisted of reciprocal parts connected by a helix. The segments were approximated by stripping the appliance at the midline in a V-shaped manner and the force was exerted by the extraoral elastics. The procedure gave results in 8 weeks, which may be regarded as a reasonable duration. The anterior cleft gap, which was $13 \mathrm{~mm}$ before the treatment, was reduced to $3 \mathrm{~mm}$ after the treatment by using modified PAM appliance. On a 21-month follow-up period, oral reshaping was regarded successful due to stability of the improved oral mold.

[Korean J Orthod 2021;51(6):428-434]

Key words: Appliances, Cleft lip and palate, Craniofacial anomalies, Functional
Received March 10, 2021; Revised July 14, 2021; Accepted July 21, 2021.

Corresponding author: Merve Gonca.

Assistant Professor, Department of Orthodontics, Recep Tayyip Erdogan University Faculty of Dentistry, Rize 53020, Turkey.

Tel +90-464-222-0000 e-mail mervegonca@gmail.com

How to cite this article: Gonca M, Ozel MB. A modified presurgical alveolar molding technique for treatment of cleft in Down syndrome. Korean J Orthod 2021;51:428-434.

(C) 2021 The Korean Association of Orthodontists.

This is an Open Access article distributed under the terms of the Creative Commons Attribution Non-Commercial License (http://creativecommons.org/licenses/by-nc/4.0) which permits unrestricted non-commercial use, distribution, and reproduction in any medium, provided the original work is properly cited. 


\section{INTRODUCTION}

Craniofacial clefts are extremely rare congenital deformities ${ }^{1}$ with an average incidence from 1.4 to 4.9 per 100,000 births. ${ }^{2}$ They are caused by the incomplete fusion of different embryonic prominences that are responsible for facial development during the prenatal eight weeks of life. Many factors, such as neural crest central disorganization, external pressures, oligohydramnios, amniotic bands, hematoma, and teratogens may constitute the etiology. ${ }^{3,4}$ Midline facial clefts were initially defined in 1823 by Bechard that result from the incomplete fusion of the medial nasal processes that form the intermaxillary segment. ${ }^{5}$

The widely accepted classification system of craniofacial clefts was proposed by Tessier. Tessier's midline cleft is termed No. 0 and may affect all the facial structures along the midline, including the upper lip, premaxilla, and nose. ${ }^{6}$

Performing the presurgical alveolar molding (PAM) or presurgical nasoalveolar molding (PNAM) in the neonatal period presents a major advantage by increasing the effectiveness of surgery that facilitates the cleft closure and nasal reshaping. Although studies concerning PAM/ PNAM treatment in infants with unilateral or bilateral cleft lip and palate have been reported, however, an extensive literature research revealed no studies regarding PAM treatment in infants with midline clefts. ${ }^{7-11}$ Thus, the purpose of this case presentation was to report the effects of a modified PAM appliance used in the treatment of an infant with a Tessier No. 0 cleft and to provide an alternative approach for shaping cleft lines in these patients.

\section{DIAGNOSIS AND ETIOLOGY}

A 7-day-old patient with Down syndrome (Trisomy 21) and midline cleft was referred to our clinic with difficulty in feeding. He was being fed through a nasogastric probe until the referral. The family history did not indicate clefts or consanguineous marriage, which might have been a probable cause for the cleft. The mother was the primary source for the detailed medical history of the infant. She reported no substance abuse (e.g., smoking, alcohol, drugs, medications) before or during her pregnancy. The infant was a third male child in the family. He had failed the hearing test. Furthermore, intraoral and extraoral examinations of the infant revealed an absence of the premaxillary bone, caudal septum, prolabium, and the columella (Figure 1) confirming the diagnosis of Tessier No. 0 cleft. A written informed consent was obtained from the patient for the publication of this case report.

\section{TREATMENT OBJECTIVES}

Our objectives were as follows: a) to eliminate or reduce the risk of aspiration while feeding by providing a separation between the oral and nasal cavity, and b) to facilitate the surgical procedure by approximating the palatal segments.

\section{TREATMENT ALTERNATIVES}

There are two different approaches for the treatment of infants with cleft lip and palate. The first approach is waiting for the growth of the infant for operation and the second approach is to reshape the lip, the palate, and the nose to facilitate the surgical procedure. The second approach is considered better than the first approach to the infant's benefit."

\section{TREATMENT PROGRESS}

An oral impression (Figure 2A) with type C siliconbased impression material (Zhermack zetaplus - putty and light body) was made in a clinical setting where an airway emergency could be handled. A stone cast was formed based on the impression (Figure 2B). A $0.8 \mathrm{~mm}$ stainless steel helix was attached to the posterior end of the appliance. Extra caution was exercised regarding the position and dimension of the helix so as not to disturb the infant's palate. After the undercuts on the stone cast were sealed with wax, an acrylic appliance was fabricated with the arms of the helix within the body of acrylic, using cold cured acrylic (imicryl-0 80 orthodontic acrylic). An acrylic button was formed on the anterior aspect of the appliance (Figure $2 \mathrm{C}$ ). The planned

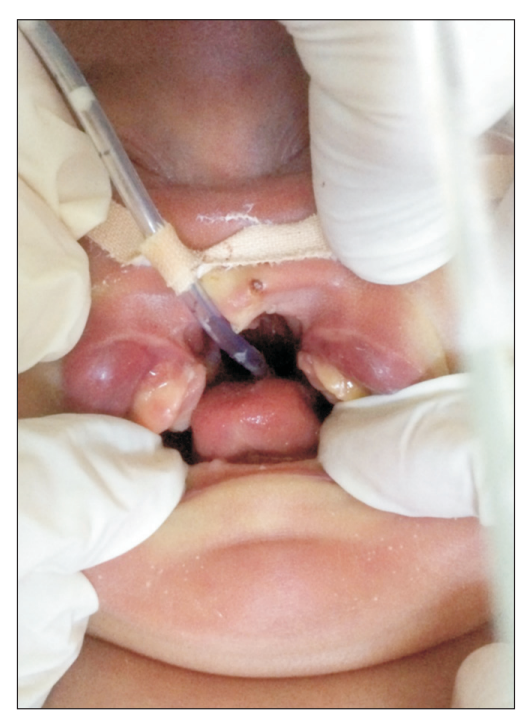

Figure 1. Pretreatment photograph. 

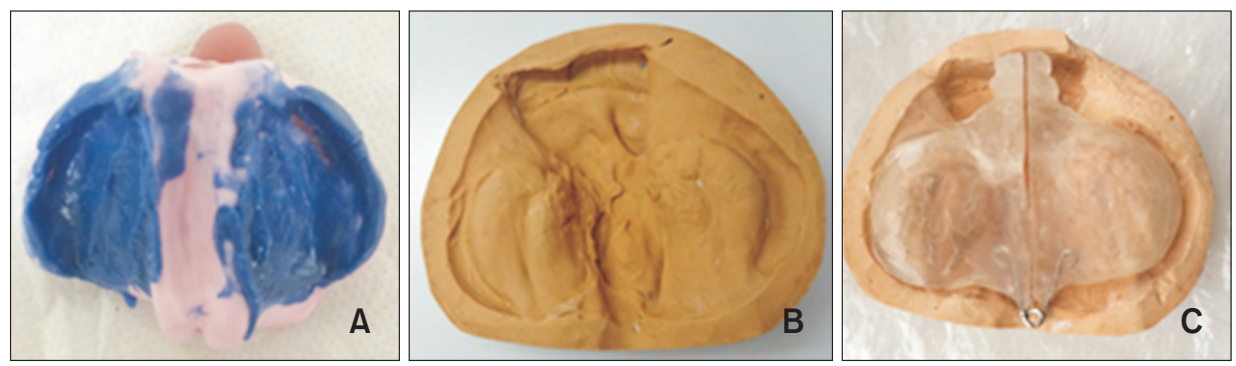

Figure 2. A, Polyvinyl siloxane impression of the infant's maxilla. B, Stone cast. C, Modified presurgical alveolar molding appliance.
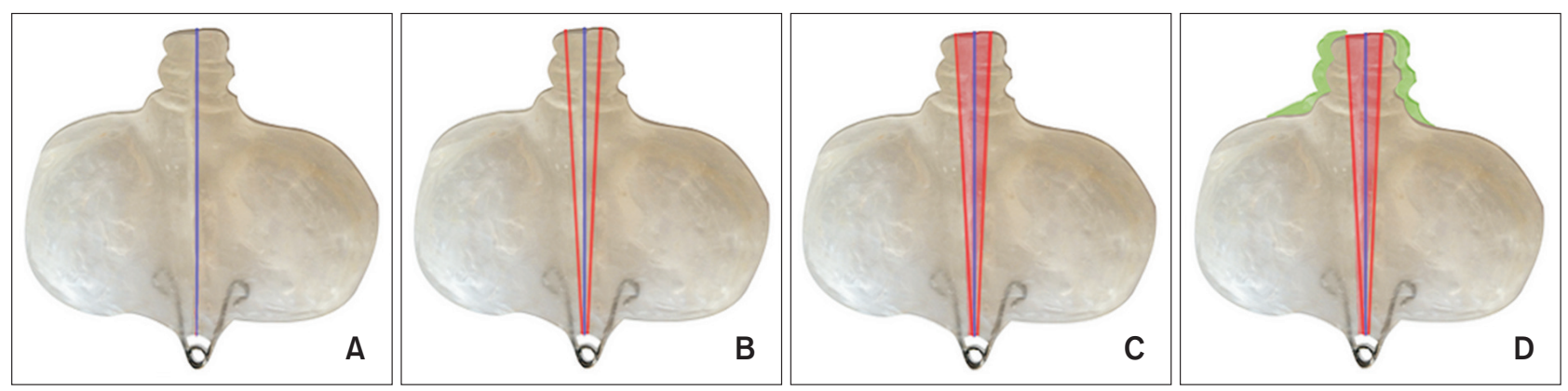

Figure 3. Procedure of the modified presurgical alveolar molding (PAM) appliance. A, The purple line demonstrates that PAM appliance consists of two reciprocal parts. $\mathbf{B}$, The red line demonstrates the boundaries of the acrylic area to be stripped. C, The red area demonstrates the acrylic region that has been removed. $\mathbf{D}$, The green area demonstrates the acrylic region where additions were made.
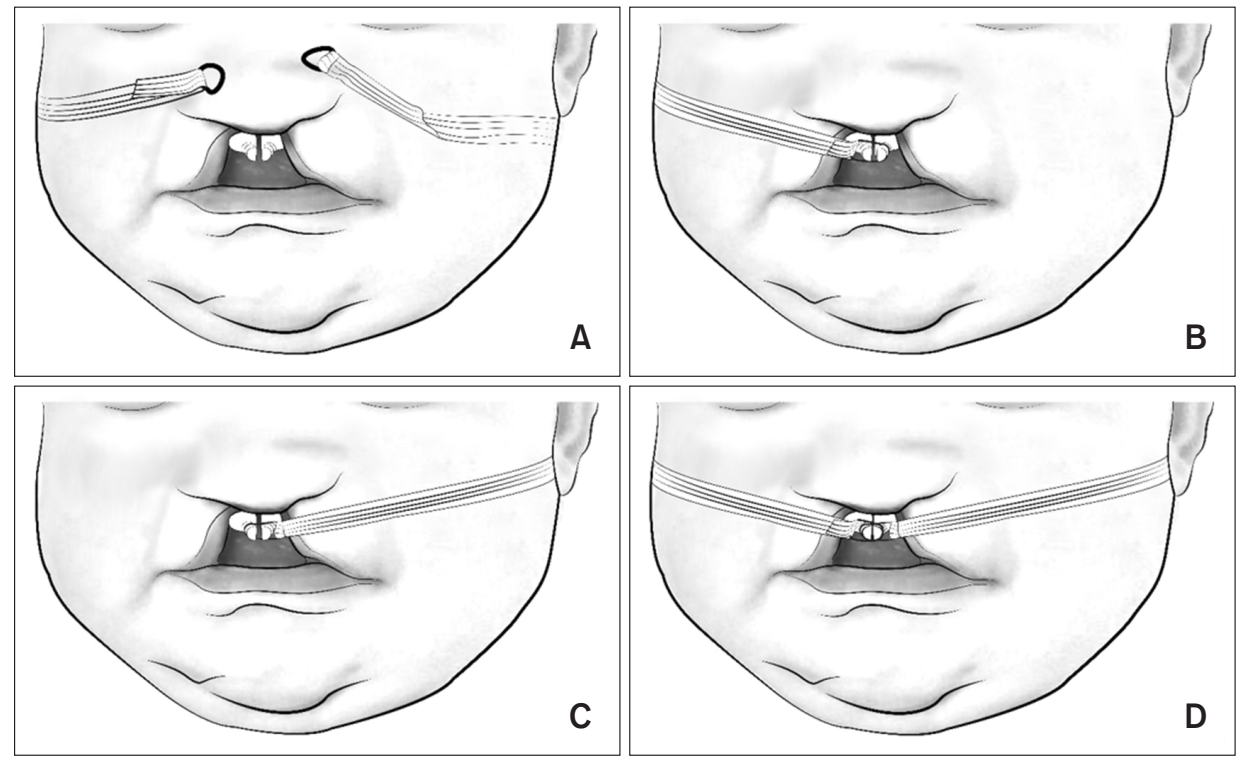

Figure 4. Demonstration of the activation of the modified presurgical alveolar molding (PAM) appliance. A, Passive state of the modified PAM appliance after placement. B, Application of the force provided by right extraoral orthodontic elastic to the left surface of the button of modified PAM appliance. C, Application of the force provided by left extraoral orthodontic elastic to the right surface of the button of modified PAM appliance. D, Approximation of the two separate parts of the modified PAM appliance with the forces provided by orthodontic elastics.

treatment procedure is demonstrated in Figure 3 and 4. The helix connected the right and left segments of the appliance including the button (Figure 3A). The inner part of the acrylic appliance, including the button, was designated for the removal process by stripping it from both the right and left segments of the appliance (Figure 
3B). The midline surfaces of the appliance were trimmed from the right and left segments in a V-shape manner at each visit (Figure 3C). The stripping process included $0.5-1 \mathrm{~mm}$ reduction in the posterior part that was closest to the helix and 1.5-2 $\mathrm{mm}$ in the anterior part of the appliance. Acrylic in the midpalatal part of the appliance was removed to create a $\mathrm{V}$-shaped opening in the passive state of the modified PAM appliance. The infant's weekly follow-ups allowed for the necessary observation of progress (Figure $3 \mathrm{~B}$ and $3 \mathrm{C}$ ). The final step of the treatment required addition of acrylic to strengthen and reshape the appliance for balanced retention of the lateral sides of the button because the selective removal procedure reduced the acrylic button size (Figure 3D). Extraoral orthodontic elastics (American orthodontics latex elastics, 3/16 inch, 61/2 oz) and sterilized strip bands (3M Steril-Strip $6 \mathrm{~mm} \times 100 \mathrm{~mm}$ ) were used on both sides to allow for oral retention of the appliance, as well as sealing the cleft gap (Figure 4). The infant's mother was instructed to replace the elastics and the bands daily.

\section{TREATMENT RESULTS}

The infant demonstrated poor adaptation during the initial phase, which might have been caused by unsuccessful parent cooperation that was later improved by parent-specialist communication. Two months after the initiation of the treatment, the anterior cleft gap was reduced from $13 \mathrm{~mm}$ to $3 \mathrm{~mm}$, as a result of successful compliance (Figure 5). The gap between the right and left segments of the palate was substantially closed. Another outcome of the modified PAM appliance use was provision of a separation between the oral and the nasal cavity that reduced food aspiration risk. After the cleft gap was reduced, we tried to reshape the nostrils with Grayson's PNAM technique used for bilateral cleft lip and palate in our patient. The nasal molding attempt failed due to a lack of compliance, and the nasal reshaping process was postponed until the surgical closure of the lip. The lip surgery was performed when the infant was 4 months (Figure 6). Nostril retainers (Koken) reshaped the nostrils following lip surgery, but unfortunately, parent cooperation could not be obtained on the second attempt too. The palate closure surgery was performed when the infant was 9 months. The infant successfully passed the hearing test performed after the palate surgery. With resultant stability of the changes seen after a follow-up period of 21 months demonstrated that oral reshaping with the modified PAM appliance was successful (Figure 7).
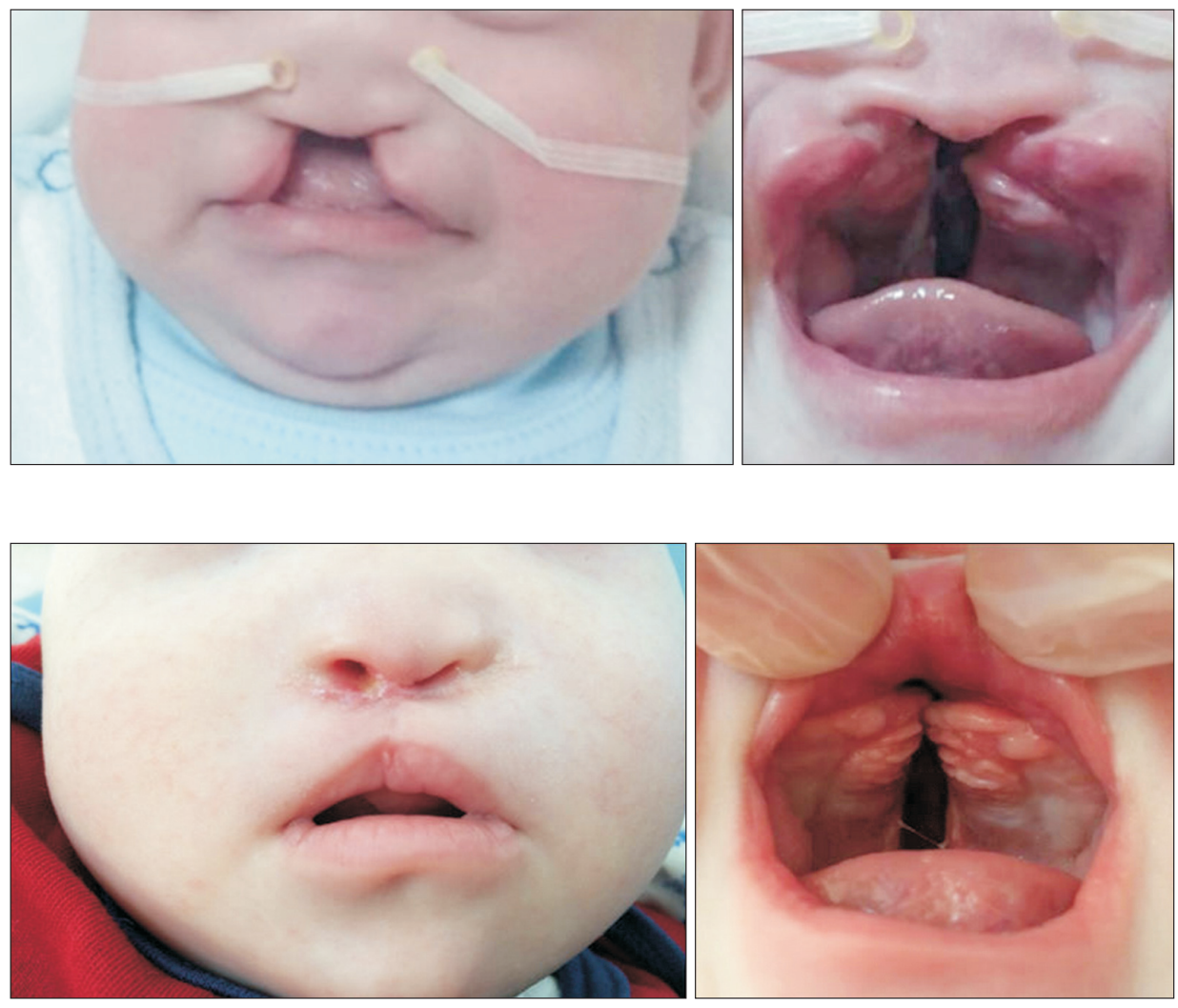

Figure 5. Two months after modified presurgical alveolar molding appliance therapy.

Figure 6. After lip surgery. 


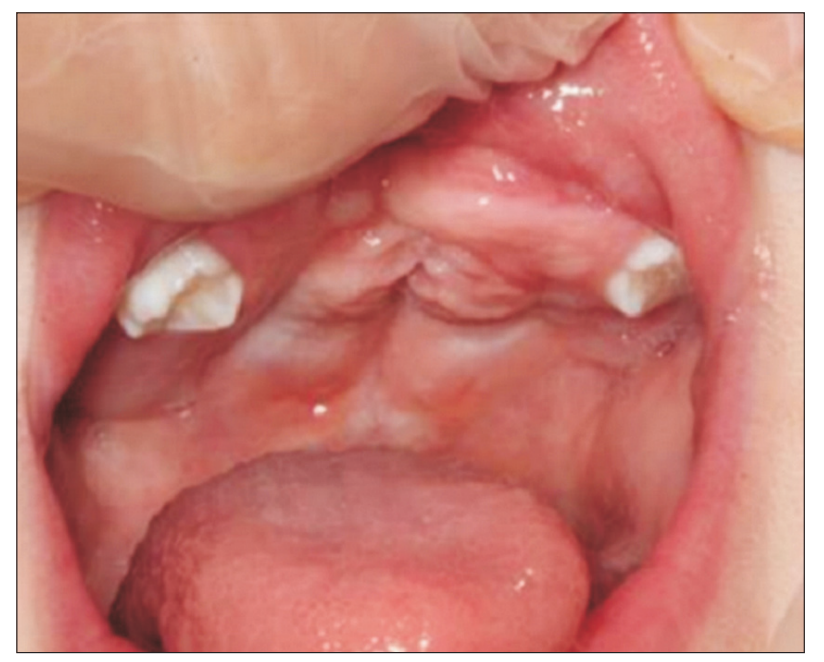

Figure 7. Twenty-one months after modified presurgical alveolar molding appliance therapy.

\section{DISCUSSION}

Cleft lip and palate might be syndromic or non-syndromic. In this case, the cleft lip and palate were associated with Down syndrome and a Tessier No. 0 cleft. The maxillary and nasal deformities were more severe for a Tessier No. 0; therefore, it was expected that the treatment would represent difficulties, resulting in compromised results.

It is essential to provide immediate treatment to infants with cleft lip and palate as early as possible due to the higher plasticity of the cartilage at birth which is caused by the high maternal estrogen in the infant's first 6-8 weeks, this provides an easier reshaping process for the tissues. ${ }^{12}$ The treatment objective is to aid the reshaping of the alveolar segments. ${ }^{7-10,13-15}$ The infant presented in this report had missing premaxillary bone, caudal septum, prolabium, and columella. Modified PAM appliance was used to reduce the gap between the alveolar segments of the maxilla in infant. In the passive state of the modified PAM appliance, there was a V-shaped opening between the segments of the appliance. Stripping was performed in a progressive manner from the posterior to the anterior surfaces. The acrylic stripping in our protocol assisted in the approximation of the palatal segments that resulted from the transmission of forces to the two-piece modified PAM appliance through the use of extraoral orthodontic elastics. One of the greatest challenges in stripping was the formation of premature contacts on the surfaces of the left and right segments that faced each other. Additional acrylic was required to supplement the lateral sides of the button to prevent it from breaking.

The mechanism of the modified PAM appliance and the fan type screw appliance function may resemble each other. The main difference between the mechanisms is the reverse activation of the fan type screw (the force applied when the screw was reversed and the gap was reduced) through the extraoral orthodontic elastics. The sterile strip band that comes from the right cheek area pulled the left side of the appliance by the extraoral orthodontic elastics and the strip from the left side of the patient's cheek pulled the right part of the modified PAM appliance; thus, this system enables to approximate the two separated parts of the appliance to come together by the forces that are transmitted by the orthodontic elastics. While the force was applied to the two parts of the modified PAM appliance through the extraoral elastics to reduce the cleft gap, the modified PAM appliance was secured in the patient's oral cavity employing a sterilized strip band to the cheeks.

Examples of different methods include several perspectives on the PAM treatment. McNeil ${ }^{13}$ used the serial appliance method to align the alveolar segments. Hotz and Gnoinski ${ }^{15}$ performed a technique involving the selective removal of acrylic from a passive acrylic appliance to guide the palatal growth and decrease the cleft gap. Georgiade and Latham ${ }^{14}$ designed a pin-retained active appliance to pull the premaxilla backwards, while widening the posterior segments for infants with bilateral cleft lip and palate. However, certain drawbacks were presented in the literature by researchers on the use of the Latham appliance system, including the high cost of the system, the requirement of sedation due to its invasive nature, and inability to focus on nasal cartilage deformities while using this system. ${ }^{11}$ The modified PAM technique is a specific noninvasive procedure presented as a hybrid of the best features of Grayson's and Latham's techniques for alveolar molding. This procedure combines Grayson's approach to acrylic addition and remov$\mathrm{al}$, and Latham's active force application during the procedure. The purpose of acrylic removal in the modified PAM procedure is to reduce the cleft gap by assisting the palate's growth towards the area that the acrylic was removed, as underlined and applied in Grayson's procedure. The purpose of adding acrylic in the modified PAM procedure is to support the button's lateral sides to prevent it from breaking. The modified PAM appliance differs from the appliance used in Grayson's procedure by the method of force application and is similar to the one used in Latham's procedure that utilizes active force generation with extraoral elastics. Another distinct feature of this procedure is non-invasiveness, like Grayson, whereas the Latham procedure is invasive.

Further development of these methods included lip and nose reshaping. Grayson ${ }^{7}$ is credited for the term "PNAM" for this procedure. Its objective is to provide a better start for surgery by reshaping the lip, palate, 
and nose. In the PNAM method, reshaping is achieved through the removal of the hard acrylic from the acrylic appliance in the direction where movement is desired while integrating the soft denture base material in the opposite direction. Furthermore, the nose is also shaped after cleft segments are approximated together, which differentiated this approach from previous methods. ${ }^{7-10}$ Before the lip surgery, Grayson's PNAM technique for bilateral cleft lip and palate was applied to reshape nostrils in our patient. However, due to lack of cooperation, the nasal reshaping process was postponed until the surgical closure of the lip. Nostril retainers (Koken) were applied after lip surgery. In the literature, another innovative application of the PNAM method is where the appliance is based on a computerized program. This method allows for a more flexible monitoring schedule for both physicians and patients by extending the intra-appointment duration. ${ }^{16}$ Generally, patients are monitored through weekly follow-ups. ${ }^{7-10}$ The computerized PNAM method allows more parent engagement in the process by transferring the responsibility of replacing the appliances in certain periods to parents because regular clinical visits might present challenges and hardships both physically and financially for the patient. ${ }^{16}$ Various researchers described extraoral nasal reshaping methods such as DynaCleft or extraoral nasal molding, separate from the oral appliance. ${ }^{17,18}$ In our patient, after the alveolar segments and the lips were brought closer, nasal reshaping procedure was attempted to reshape the nostrils as the infant lacked a premaxillary bone, caudal septum, prolabium, and columella. Successful nasal molding using Grayson's nasal stent and nostril retainer could not be performed in our patient due to cooperation issues with the parents.

The anterior cleft gap, which was $13 \mathrm{~mm}$ before the treatment, was reduced to $3 \mathrm{~mm}$ after the treatment, and the lips were brought closer. Contrary to a randomized controlled clinical study concluding that alveolar reshaping was not necessary before surgery with regards to long-term effects on dentoalveolar structures, ${ }^{19}$ the successful immediate and 21-month follow-up clinical outcome with the approach presented herein may favor studies reporting a positive effect of PAM on dentoalveolar structures. ${ }^{7-10}$

\section{CONCLUSION}

Individual modifications and adjustments may be required in addition to routine procedures in line with the specific needs of special situations such as Tessier No. 0. This approach provides increased comfort and an effective process in reducing cleft gaps for infants.

\section{CONFLICTS OF INTEREST}

No potential conflict of interest relevant to this article was reported.

\section{REFERENCES}

1. Kawamoto HH. Rare craniofacial clefts. In: McCarthy JG, ed. Plastic surgery. Philadelphia: W.B. Saunders; 1990. p. 2952-5.

2. Ozaki W, Kawamoto HK Jr. Craniofacial clefting. In: Lin K, Ogle R, Jane J, eds. Craniofacial surgery: science and surgical technique. Philadelphia: W.B. Saunders; 2002. p. 309-31.

3. Rintala AE. Epidemiology of orofacial clefts in Finland: a review. Ann Plast Surg 1986;17:456-9.

4. Starck WJ, Epker BN. Surgical repair of a median cleft of the upper lip. J Oral Maxillofac Surg 1994;52:1217-9.

5. Kawamoto HK, Patel PK. Atypical facial clefts. In: Bentz M, ed. Pediatric plastic surgery. Stamford: Appleton \&t Lange, cop.; 1998. p. 175-225.

6. Tessier P. Anatomical classification facial, craniofacial and latero-facial clefts. J Maxillofac Surg 1976;4:69-92.

7. Grayson BH, Santiago PE, Brecht LE, Cutting CB. Presurgical nasoalveolar molding in infants with cleft lip and palate. Cleft Palate Craniofac J 1999;36:486-98.

8. Grayson BH, Cutting CB. Presurgical nasoalveolar orthopedic molding in primary correction of the nose, lip, and alveolus of infants born with unilateral and bilateral clefts. Cleft Palate Craniofac J 2001;38:193-8.

9. Grayson BH, Maull D. Nasoalveolar molding for infants born with clefts of the lip, alveolus, and palate. Semin Plast Surg 2005;19:294-301.

10. Grayson BH, Garfinkle JS. Nasoalveolar molding and columella elongation in preparation for the primary repair of unilateral and bilateral cleft lip and palate. In: Losee JE, Kirschner RE, eds. Comprehensive cleft care. New York: McGraw-Hill Medical; 2009. p. 70120.

11. Grayson BH, Garfinkle JS. Early cleft management: the case for nasoalveolar molding. Am J Orthod Dentofacial Orthop 2014;145:134-42.

12. Matsuo K, Hirose T, Tomono T, Iwasawa M, Katohda $\mathrm{S}$, Takahashi N, et al. Nonsurgical correction of congenital auricular deformities in the early neonate: a preliminary report. Plast Reconstr Surg 1984;73:3851.

13. McNeil CK. Orthodontic procedures in the treatment of congenital cleft palate. Dent Rec (London) 1950;70:126-32. 
14. Georgiade NG, Latham RA. Maxillary arch alignment in the bilateral cleft lip and palate infant, using pinned coaxial screw appliance. Plast Reconstr Surg 1975;56:52-60.

15. Hotz M, Gnoinski W. Comprehensive care of cleft lip and palate children at Zürich university: a preliminary report. Am J Orthod 1976;70:481-504.

16. Gong X, Yu Q. Correction of maxillary deformity in infants with bilateral cleft lip and palate using computer-assisted design. Oral Surg Oral Med Oral Pathol Oral Radiol 2012;114(5 Suppl):S74-8.

17. Doruk C, Kiliç B. Extraoral nasal molding in a newborn with unilateral cleft lip and palate: a case re- port. Cleft Palate Craniofac J 2005;42:699-702.

18. Monasterio L, Ford A, Gutiérrez C, Tastets ME, García J. Comparative study of nasoalveolar molding methods: nasal elevator plus DynaCleft ${ }^{\circledR}$ versus NAM-Grayson in patients with complete unilateral cleft lip and palate. Cleft Palate Craniofac J 2013;50:548-54.

19. Bongaarts CA, van't Hof MA, Prahl-Andersen B, Dirks IV, Kuijpers-Jagtman AM. Infant orthopedics has no effect on maxillary arch dimensions in the deciduous dentition of children with complete unilateral cleft lip and palate (Dutchcleft). Cleft Palate Craniofac J 2006;43:665-72. 